\title{
Chalcogels as electrode materials for Li-ion batteries
}

\author{
Vicky V. T. Doan-Nguyen, ${ }^{*, \dagger},+$ Kota S. Subrahmanyam, "Megan M. Butala, ${ }^{\S}$ \\ Jeffrey A. Gerbec," Saiful M. Islam, ${ }^{\top}$ Katherine N. Kanipe, ${ }^{\perp}$ Catrina E. Wilson, ${ }^{\perp}$ \\ Mahalingam Balasubramanian,\# Kamila M. Wiaderek,\# Olaf J. Borkiewicz,\# \\ Karena W. Chapman, \# Peter J. Chupas, ${ }^{\#}$ Martin Moskovits, ${ }^{\perp}$ Bruce S. Dunn, ${ }^{@}$ \\ Mercouri G. Kanatzidis, "and Ram Seshadri ${ }^{\ddagger},, \perp$
}

†California NanoSystems Institute, University of California, Santa Barbara, CA 93106 $\ddagger$ Materials Research Laboratory, University of California, Santa Barbara, CA 93106 \Department of Chemistry, Northwestern University, Evanston, IL 60208 §Materials Department, University of California, Santa Barbara, CA 93106 ||Mitsubishi Chemical Center for Advanced Materials, Santa Barbara, CA 93106 $\perp$ Department of Chemistry and Biochemistry, University of California, Santa Barbara, CA 93106 \#X-ray Science Division, Advanced Photon Source, Argonne National Laboratory, Argonne, IL 60439 @Department of Materials Science and Engineering, University of California, Los Angeles, CA 90095 E-mail: vdn@mrl.ucsb.edu 


\begin{abstract}
Sulfur cathodes in conversion reaction batteries operate by a different mechanism than that of intercalation materials, and they possess larger theoretical capacities. However, sulfur-based electrode materials suffer from parasitic polysulfide shuttling, which contribute to decreased capacity retention and cyclability. We demonstrate that transition metal polysulfide chalcogels achieve high gravimetric capacity as electrode materials for lithium-ion batteries. Transition metal polysulfide chalcogels are amorphous, and comprise polysulfide chains connected by inorganic linkers. The linkers appear to act as a "glue" in the electrode to prevent polysulfide shuttling. In particular, we examined a $4 \mathrm{~d}$ transition metal polysulfide chalcogel to investigate the role of the inorganic linker in maintaining structural stability in the electrode. In the $\operatorname{MoS}_{3.4}$ case study, the Mo polysulfide chalcogels function as electrodes in carbonate- as well as ether-based electrolytes and achieve an initial gravimetric capacity of $600 \mathrm{mAh}$ $\mathrm{g}^{-1}$. We employ X-ray absorption spectroscopy and operando pair distribution function techniques to elucidate the structural evolution of the electrode. Raman and X-ray photoelectron spectroscopy track the chemical moieties that arise during the anionredox-driven processes. We find the redox state of Mo remains unchanged across the electrochemical cycling even to $1 \mathrm{~V}$ and correspondingly, the redox is anion-driven. Chalcogels offer a new class of electrode materials for achieving high-capacity Li-ion batteries.
\end{abstract}

